\title{
Adoção de terapias nutricionais enteral e parenteral associada à redução da taxa de óbitos de pacientes neoplásicos
}

\author{
Adoption of enteral and parenteral nutritional therapies associated with reduced \\ death rate in neoplastic patients \\ Eduardo Botti Abbade ${ }^{1}$
}

\begin{abstract}
RESUMO
Modelo do estudo: Estudo descritivo transversal.Objetivo: Este estudo analisou a relação entre a adoção da nutrição enteral e parenteral adulta e o tempo de permanência médio, a taxa de óbitos e o custo das internações de pacientes neoplásicos em hospitais do Rio Grande do Sul. Método: Foram analisados os dados dissemináveis disponíveis no SIH/SUS de 2017 do Rio Grande do Sul específicos para internações realizadas sob o diagnóstico principal associado ao CID Cap. II (Neoplasmas). Os dados foram analisados por observação da média e teste de Kruskal Wallis. Resultados: Foi observada taxa de óbito significativamente inferior em pacientes neoplásicos que foram internados em UTI e que foram submetidos às terapias dietéticas enterais e/ou parenterais, em relação aos pacientes que não receberam tais suplementos, em casos de diagnóstico de neoplasias malignas no esôfago (C15), no intestino delgado (C17) e no pâncreas (C25). Já o tempo médio de permanência e valor total médio de internações de pacientes neoplásicos tendem a ser maior em pacientes que recebem terapias dietéticas enterais e/ou parenterais, possivelmente devido à gravidade do quadro clínico. Conclusão: A adoção de nutrição enteral e parenteral apresenta potencial significativo na redução da taxa de mortalidade de pacientes com câncer no sistema digestivo, principalmente dos casos mais graves que necessitam de internação em UTI.
\end{abstract}

Palavras-chave: Terapia Nutricional; Neoplasias; Desnutrição; Nutrição Enteral; Nutrição Parenteral.

\begin{abstract}
Study design: Cross-sectional descriptive study. Objective: This study aimed to analyze the association between the adoption of adult enteral and parenteral nutrition and the average length of hospitalization, the average mortality rate, and the average total cost for admissions of neoplastic patients in hospitals in Rio Grande do Sul. Method: The study analyzes the data available obtained at the SIH/SUS in 2017 for hospitals in Rio Grande do Sul state, for specific hospitalizations registered under the main diagnosis Neoplasms (ICD - Chapter II). Data were analyzed through the average value of the main variables as well as the Kruskal Wallis test. Results: Significantly lower death rate was observed in ICU patients who underwent enteral and/or parenteral dietary therapy than patients who did not receive such supplements in cases of malignant esophageal cancer (C15), in the small intestine (C17), and pancreas (C25). The average length of stay and the average total value of hospitalizations of neoplastic patients tend to be higher in patients receiving enteral and/or parenteral dietary therapies, possibly due to the severity of the clinical condition. Conclusions: The adoption of enteral and parenteral nutrition has significant potential to reduce the mortality rate of cancer patients in the digestive system, especially the most severe cases requiring ICU admission.
\end{abstract}

Keywords: Nutrition Therapy; Neoplasms; Malnutrition; Enteral Nutrition; Parenteral Nutrition.

1. Departamento de Ciências Administrativas, Universidade Federal de Santa Maria (UFSM), Santa Maria (RS), Brasil.

$\triangle$ Eduardo Botti Abbade. Avenida Roraima, 1000, Prédio 74C, Camobi. CEP: 97105-900. Santa Maria (RS), Brasil. eduardo.abbade@ufsm.br Recebido em: 19/08/2019 | Aprovado em: 13/03/2020 


\section{INTRODUÇÃO}

O quadro de desnutrição é frequente em internação hospitalar sendo estimado em cerca de $50 \%{ }^{1,2}$. Tal desnutrição pode comprometer a função imunológica, ocasionar deficiência de movimentação ventilatória e enfraquecer os músculos respiratórios, levando a uma possível dependência prolongada de ventiladores pulmonares, aumentando o risco de morbidade e mortalidade por infeç̧ões ${ }^{3,4}$. Além disso, durante a hospitalização pode ocorrer diminuição do apetite, diminuição na ingestão de nutrientes, e efeitos colaterais dietéticos causados pelos procedimentos médicos e intervenções medicamentosas, podendo deteriorar o quadro nutricional do paciente ${ }^{5-7}$. Logo, a desnutrição em pacientes internados implica em elevação do risco de infecções e complicações médicas, aumentando o custo da hospitalização e o risco de mortalidade ${ }^{8-10}$.

Evidências mostram que a adequada ingestão energética e proteica logo no início da internação traz grandes benefícios para o paciente e para a instituição de saúde ${ }^{11}$. Objetivando reduzir os riscos de complicações, é recomendado que seja utilizado logo no início da internação terapias de Nutrição Enteral (NE) em pacientes incapazes de fazer a ingestão nutricional adequada voluntariamente ${ }^{12,13}$. Adicionalmente, em várias situações, apenas a NE pode ser insuficiente para suprir os pacientes com as quantidades energéticas e proteicas adequadas $^{10,14,15}$, sendo que a utilização de terapias dietéticas por meio de nutrição parenteral (NP) pode ser necessária para alcançar melhores resultados ${ }^{16,17}$. No entanto, ocorrem ainda resistências por parte dos profissionais das instituições de saúde em adotar as terapias de nutrição parenteral devido ao seu elevado custo e riscos percebidos ${ }^{18,19}$.

Segundo dados da Organização Mundial da Saúde, o câncer é a segunda principal causa de morte em todo o mundo, sendo responsável por cerca de 9,6 milhões de mortes em $2018^{20}$. Ademais, o impacto econômico do câncer é significativo e apresenta tendência de crescimento. Levantamento feito pelo INCA estimou que os custos econômicos diretos do câncer no Brasil em 1999 eram de $\mathrm{R} \$ 470,05$ milhões, passando para $\mathrm{R} \$ 1.569$ bi-
Ihões em 2009, e atingindo um patamar de cerca de $\mathrm{R} \$ 3.280,25$ bilhões em $2015^{21}$. Tendo em vista a necessidade de promover estudos com potencial de elucidar melhor questões acerca dos potenciais benefícios do uso de terapias dietéticas com eventual redução dos custos e mortalidade de pacientes neoplásicos, este estudo objetivou descrever o nível de adoção da nutrição enteral e parenteral adulta e sua relação com o tempo de permanência médio, a taxa média de óbitos e o custo médio registrados nas internações de pacientes neoplásicos em hospitais do Rio Grande do Sul.

\section{MATERIAL E MÉTODO}

Foi realizado levantamento de dados extraídos do Sistema de Internações Hospitalares do Sistema Único de Saúde (SIH/SUS). Tal sistema é construído utilizando as informações provenientes das Autorizações de Internação Hospitalar (AIH), documento obrigatório que habilita as internações hospitalares realizadas no SUS. Os dados do estudo foram coletados com o auxílio do software TABWIN que permite a leitura dos arquivos dissemináveis públicos (pacotes de dados disponibilizados mensalmente pelo Ministério da Saúde) que se encontram disponíveis para download no Departamento de Informática do SUS (DATASUS). O TABWIN é o software de leitura dos dados disponibilizados pelo SIH/SUS.

Inicialmente foram obtidos os pacotes de dados mensais de internações hospitalares (arquivos $\mathrm{RD}$ ) e pacotes de dados mensais com os procedimentos realizados nas internações (arquivos SD) do ano de 2017 do estado do Rio Grande do Sul. A partir destas bases de dados mensais, foram extraídos os dados referentes às internações realizadas sob o diagnóstico principal vinculado ao CID Cap. II (neoplasias). Tais registros deram origem a uma base de dados única formada por duas tabelas (dados de internações hospitalares [RD] e dados de procedimentos principais e secundários realizados em cada internação [SP]), sendo que tais tabelas foram relacionadas através da variável de código da internação (Número da AIH). O Quadro 1 apresenta as variáveis mais relevantes que constam em cada uma das tabelas da base de dados. 


\section{Quadro 1}

Variáveis observadas nas bases de dados dissemináveis do SIH/SUS

\begin{tabular}{|l|l|}
\hline Tabela de Internações Hospitalares (RD) & Tabela de Procedimentos Realizados (SP) \\
\hline - Número da AIH; & - Número da AIH; \\
- CID do Diagnóstico Principal (C00 à D48); & - CID do Diagnóstico Principal (C00 à D48); \\
- Sexo do paciente (variável dicotômica); & - Procedimento Secundário (03.09.01.004-7 \\
- Idade do paciente em anos (12 anos ou mais); & e 03.09.01.007-1). \\
- Óbito (variável dicotômica); & \\
- Tempo de Permanência (em dias); & \\
- Valor Total da Internação (em Reais); & \\
- Valor em UTI (em Reais). & \\
\hline
\end{tabular}

Foram filtradas da tabela de procedimentos (SP) apenas as internações que utilizaram procedimentos de Nutrição Enteral em Adulto (código 03.09.01.004-7) e Nutrição Parenteral em Adulto (código 03.09.01.007-1). A Nutrição Enteral é a fórmula nutricional completa, administrada através de sondas nasoentérica, nasogástrica, de jejunostomia ou de gastrostomia. Já a Nutrição Parenteral é a fórmula administrada por via intravenosa, sendo uma solução ou emulsão composta obrigatoriamente de aminoácidos, carboidratos, vitaminas e minerais, com ou sem administração diária de lipídios para suprir as necessidades metabólicas e nutricionais de pacientes impossibilitados de alcançá-la por via oral ou enteral. Em se tratando de terapias dietéticas para adultos, os pacientes devem ter idade igual ou superior a 12 anos.

Em uma análise preliminar dos dados, o estudo identificou os seis CIDs com maior quantitativo de procedimentos de Nutrição Enteral Adulta: (1) C15 (Neoplasia maligna do esôfago); (2) C16 (Neoplasia maligna do estômago); (3) C34 (Neoplasia maligna dos brônquios e dos pulmões); (4) C71 (Neoplasia maligna do encéfalo); (5) C32 (Neoplasia maligna da laringe); e (6) C10 (Neoplasia maligna da orofaringe). Também foram identificados os seis CIDs com maior quantitativo de procedimentos de Nutrição Parenteral Adulta: (1) C18 (Neoplasia maligna do cólon); (2) C16 (Neoplasia maligna do estômago); (3) C15 (Neoplasia maligna do esôfago); (4) C17 (Neoplasia maligna do intestino delgado); (5) C25 (Neoplasia maligna do pâncreas); e (6) C20 (Neoplasia maligna do reto). Considerando os diagnósticos que apresentam maiores quantitativos de uso de procedimentos de terapia nutricional enteral e parenteral adulta, as análises foram conduzidas considerando todas as internações realizadas sob o diagnóstico principal de CID Cap. II, e considerando os grupos de internações específicos realizados sob os diagnósticos principais de C15, C16, C34, C71, C32, C10, C18, C25, C17 e C20.

As internações foram categorizadas de acordo com o uso de terapias dietéticas, sendo: (1) nenhum uso; (2) apenas nutrição enteral; (3) apenas nutrição parenteral; e (4) nutrição enteral e parenteral. Os dados referentes à permanência média, taxa média de óbitos e valor médio das internações para os CIDs selecionados foram analisados utilizando estatística descritiva (média aritmética) e teste não-paramétrico de Kruskal Wallis, este indicado para efetuar comparações entre duas ou mais amostras independentes de tamanhos iguais ou diferentes. Os resultados das médias obtidas para as quatro categorias de uso de terapias dietéticas foram analisados de forma interpretativa, destacando-se a identificação de redução observada nos grupos que adotam algum tipo de terapia dietética (enteral e/ou parenteral). Na sequência, os dados foram submetidos ao teste de Kruskal Wallis de modo a consubstanciar os resultados verificados preliminarmente por meio da análise das médias aritméticas.

\section{RESULTADOS}

A análise inicial da base de dados com 64.341 internações registradas no ano de 2017 em hospitais do Rio Grande do Sul revelou que o perfil dos pacientes internados via SUS sob o diagnóstico principal de CID Cap. II (Neoplasias) é de 53,7\% do sexo feminino e a média de idade geral é de 56,2 anos. A idade média dos pacientes do sexo feminino, total de 34.542 internações, é de 54,6 anos. Já a idade média dos pacientes do sexo masculino, total de 29.799 internações, é de 58,1 anos. O tempo médio 
de permanência geral das internações é de 6,18 dias e a taxa de óbitos geral é de $9 \%$. Para os pacientes do sexo masculino o tempo médio de permanência é de 6,95 dias e a taxa de óbitos é de $11 \%$. Já para os pacientes do sexo feminino o tempo médio de permanência é de 5,51 dias e a taxa de óbitos é de $8 \%$.

No ano de 2017, conforme base de dados do estudo, foram aprovados via SUS 2.258.739 ( $R \$ 75.307 .746,00)$ procedimentos de Nutrição Enteral em Adultos (Cód. 0309010047) e 135.857 ( $R \$ 8.892 .105,00)$ procedimentos de Nutrição $\mathrm{Pa}$ renteral em Adultos (Cód. 0309010071), evidenciando que o uso de terapia de nutrição enteral é expressivamente superior ao uso de terapia de nutrição parenteral em pacientes internados via SUS. As internações sob o diagnóstico principal associadas ao CID Cap. II [Neoplasias (tumores)] foram responsáveis por 271.517 (R\$ 8.322.412,50) procedimentos de Nutrição Enteral Adulta e por 32.772 ( $\$ \$ 2.008 .023,00)$ procedimentos de Nutrição Parenteral Adulta em 2017 , representando $12,02 \%$ e $24,12 \%$ do total de procedimentos de Nutrição Enteral e Parenteral, respectivamente. Dentre os CIDs do Cap. II, - CID responsável pela maior parte dos procedimentos de Nutrição Enteral Adulta aprovados via SUS no Brasil foi o CID-C15 (Neoplasia maligna do esôfago), com 29.904.
Os hospitais do estado do Rio Grande do Sul (RS) foram responsáveis por $11,45 \%$ (qtd. 258.666; R\$ 7.758.780,00) dos procedimentos de Nutrição Enteral Adulta e 11,86\% (qtd. 16.115; R\$ $966.900,00$ ) dos procedimentos de Nutrição Parenteral Adulta administrados em pacientes internados via SUS do Brasil. As internações sob o diagnóstico principal associadas ao CID Cap. II [Neoplasias [tumores)] registradas no RS foram responsáveis por $36.320(\mathrm{R} \$ 1.089 .600,00)$ procedimentos de Nutrição Enteral Adulta e por 3.582 $(R \$ 214.920,00)$ procedimentos de Nutrição Parenteral Adulta em 2017, representando $14,04 \%$ e $22,23 \%$ do total de procedimentos de Nutrição Enteral e Parenteral, respectivamente registrados nos hospitais do RS. Dentre os CIDs do Cap. II, o CID responsável pela maior parte dos procedimentos de Nutrição Enteral Adulta aprovados via SUS no RS foi o CID-15 (Neoplasia maligna do esôfago) com 5.598.

A Tabela 1 mostra os resultados obtidos para as análises específicas acerca da avaliação comparativa do tempo médio de permanência, taxa média de óbitos e valor médio da internação para as internações que não utilizaram nenhum tipo de terapia dietética, as internações que utilizaram apenas a Nutrição Enteral (NE), as internações que utilizaram apenas a Nutrição Parenteral (NP) e as internações que utilizaram ambas as nutrições Enteral e Parenteral (NEP).

\section{Tabela 1}

Relação entre Indicadores Hospitalares (tempo de permanência, taxa de óbito e valor médio da internação) e o uso de Terapias Nutricionais Enterais e Parenterais em pacientes neoplásicos com idade igual ou superior a 12 anos, de hospitais públicos do RS em 2017

\begin{tabular}{|c|c|c|c|c|c|c|c|}
\hline \multirow{2}{*}{ CID } & \multirow{2}{*}{ Variável } & \multicolumn{4}{|c|}{ Terapia Dietética Utilizada } & \multirow{2}{*}{ Análise geral } & \multirow{2}{*}{ Kruskal-Wallis ANOVA } \\
\hline & & Nenhuma & NE & NP & NEP & & \\
\hline \multirow{4}{*}{$\begin{array}{l}\boxminus \\
\stackrel{0}{0} \\
\stackrel{0}{0} \\
\stackrel{0}{U}\end{array}$} & $\begin{array}{l}\text { Permanência } \\
\text { Média (dias) }\end{array}$ & 5,5 & 13,33 & 20,08 & 26,15 & aumento & $K W=3991,28 ; p<0,0001$ \\
\hline & $\begin{array}{l}\text { Taxa Média de } \\
\text { Óbitos }\end{array}$ & 0,0802 & 0,2805 & 0,2029 & 0,2182 & aumento & $K W=1907 ; p<0,0001$ \\
\hline & $\begin{array}{l}\text { Valor Médio } \\
\text { das AIHs }\end{array}$ & 1820,77 & 3620,06 & 7427,57 & 8850,97 & aumento & $K W=1983,99 ; p<0,0001$ \\
\hline & Qtd. AIHs & 57710 & 4249 & 276 & 55 & - & - \\
\hline \multirow{4}{*}{$\begin{array}{l}\stackrel{\ln }{u} \\
0 \\
0\end{array}$} & $\begin{array}{l}\text { Permanência } \\
\text { Média (dias) }\end{array}$ & 5,74 & 10,24 & 18,95 & 38,67 & aumento & $K W=226,97 ; p<0,0001$ \\
\hline & $\begin{array}{l}\text { Taxa Média de } \\
\text { Óbitos }\end{array}$ & 0,1563 & 0,0990 & 0,2273 & 0,3333 & $\begin{array}{l}\text { redução } \\
\text { observada }\end{array}$ & $K W=18,53 ; p=0,0003$ \\
\hline & $\begin{array}{l}\text { Valor Médio } \\
\text { das AIHs }\end{array}$ & 1132,89 & 2621,62 & 9141,4 & 9110,58 & aumento & $\mathrm{KW}=370,71 ; p<0,0001$ \\
\hline & Qtd. AIHs & 1459 & 727 & 22 & 12 & - & - \\
\hline
\end{tabular}


Tabela 1 (Continuação)

\begin{tabular}{|c|c|c|c|c|c|c|c|}
\hline \multirow{2}{*}{ CID } & \multirow{2}{*}{ Variável } & \multicolumn{4}{|c|}{ Terapia Dietética Utilizada } & \multirow{2}{*}{ Análise geral } & \multirow{2}{*}{ Kruskal-Wallis ANOVA } \\
\hline & & Nenhuma & NE & NP & NEP & & \\
\hline \multirow{4}{*}{$\begin{array}{l}\bullet \\
\cup \\
\cup \\
0 \\
\ddots\end{array}$} & $\begin{array}{l}\text { Permanência } \\
\text { Média (dias) }\end{array}$ & 5,9 & 11,17 & 20,5 & 16,33 & aumento & $K W=210,38 ; p<0,0001$ \\
\hline & $\begin{array}{l}\text { Taxa Média de } \\
\text { Óbitos }\end{array}$ & 0,1335 & 0,163 & 0,1471 & 0,1667 & aumento & $K W=2,39 ; p=0,4951$ \\
\hline & $\begin{array}{l}\text { Valor Médio } \\
\text { das AIHs }\end{array}$ & 1585,11 & 3819,88 & 5775,21 & 7947,85 & aumento & $K W=267,13 ; p<0,0001$ \\
\hline & Qtd. AIHs & 1596 & 405 & 34 & 6 & - & - \\
\hline \multirow{4}{*}{$\begin{array}{l}\stackrel{J}{U} \\
\text { 光 }\end{array}$} & $\begin{array}{l}\text { Permanência } \\
\text { Média (dias) }\end{array}$ & 8,1 & 13,66 & 29 & - & aumento & $K W=89,27 ; p<0,0001$ \\
\hline & $\begin{array}{l}\text { Taxa Média de } \\
\text { Óbitos }\end{array}$ & 0,1781 & 0,5277 & 1 & - & aumento & $K W=192,44 ; p<0,0001$ \\
\hline & $\begin{array}{l}\text { Valor Médio } \\
\text { das AIHs }\end{array}$ & 1592,48 & 2585,4 & 12925,84 & - & aumento & $K W=74,32 ; p<0,0001$ \\
\hline & Qtd. AIHs & 3335 & 271 & 1 & - & - & - \\
\hline \multirow{4}{*}{$\begin{array}{l}\stackrel{-}{U} \\
0 \\
0\end{array}$} & $\begin{array}{l}\text { Permanência } \\
\text { Média (dias) }\end{array}$ & 9,84 & 13,32 & 1 & - & aumento & $K W=27,44 ; p<0,0001$ \\
\hline & $\begin{array}{l}\text { Taxa Média de } \\
\text { Óbitos }\end{array}$ & 0,1109 & 0,3368 & 0 & - & aumento* & $K W=63,81 ; p<0,0001$ \\
\hline & $\begin{array}{l}\text { Valor Médio } \\
\text { das AIHs }\end{array}$ & 2635,72 & 5313,46 & 2066,9 & - & aumento* & $K W=73,22 ; p<0,0001$ \\
\hline & Qtd. AIHs & 938 & 190 & 1 & - & - & - \\
\hline \multirow{4}{*}{$\begin{array}{l}\stackrel{N}{U} \\
\text { Oㅂㅂ }\end{array}$} & $\begin{array}{l}\text { Permanência } \\
\text { Média (dias) }\end{array}$ & 4,81 & 10,08 & - & 19,5 & aumento & $K W=133,58 ; p<0,0001$ \\
\hline & $\begin{array}{l}\text { Taxa Média de } \\
\text { Óbitos }\end{array}$ & 0,0881 & 0,1013 & - & 0 & aumento* & $K W=0,63 ; p=0,7294$ \\
\hline & $\begin{array}{l}\text { Valor Médio } \\
\text { das AIHs }\end{array}$ & 1229,59 & 2848,14 & - & 2997,36 & aumento & $\mathrm{KW}=79,74 ; p<0,0001$ \\
\hline & Qtd. AIHs & 590 & 227 & - & 2 & - & - \\
\hline \multirow{4}{*}{$\begin{array}{l}\stackrel{ }{U} \\
\text { U } \\
\text { 븐 }\end{array}$} & $\begin{array}{l}\text { Permanência } \\
\text { Média (dias) }\end{array}$ & 5,58 & 13,98 & 8 & 16 & aumento & $K W=69,75 ; p<0,0001$ \\
\hline & $\begin{array}{l}\text { Taxa Média de } \\
\text { Óbitos }\end{array}$ & 0,0974 & 0,1958 & 0 & 1 & aumento* & $K W=14,42 ; p=0,0024$ \\
\hline & $\begin{array}{l}\text { Valor Médio } \\
\text { das AIHs }\end{array}$ & 1197,5 & 2174,15 & 1904,63 & 10189,37 & aumento & $K W=23,38 ; p<0,0001$ \\
\hline & Qtd. AIHs & 267 & 143 & 1 & 1 & - & - \\
\hline \multirow{4}{*}{$\begin{array}{l}\stackrel{\infty}{U} \\
0 \\
\ddots\end{array}$} & $\begin{array}{l}\text { Permanência } \\
\text { Média (dias) }\end{array}$ & 5,58 & 15,46 & 19,16 & 18,5 & aumento & $K W=315,63 ; p<0,0001$ \\
\hline & $\begin{array}{l}\text { Taxa Média de } \\
\text { Óbitos }\end{array}$ & 0,0674 & 0,3277 & 0,254 & 0,125 & aumento & $K W=185,58 ; p<0,0001$ \\
\hline & $\begin{array}{l}\text { Valor Médio } \\
\text { das AIHs }\end{array}$ & 2016,74 & 4153,69 & 7940,65 & 9209,94 & aumento & $K W=242,53 ; p<0,0001$ \\
\hline & Qtd. AIHs & 4705 & 177 & 63 & 8 & - & - \\
\hline \multirow{4}{*}{$\begin{array}{l}\stackrel{\operatorname{Un}}{U} \\
0 \\
\stackrel{U}{U}\end{array}$} & $\begin{array}{l}\text { Permanência } \\
\text { Média (dias) }\end{array}$ & 8,08 & 16,26 & 20,19 & 13 & aumento & $K W=81,31 ; p<0,0001$ \\
\hline & $\begin{array}{l}\text { Taxa Média de } \\
\text { Óbitos }\end{array}$ & 0,2914 & 0,3016 & 0,1429 & 0 & $\begin{array}{l}\text { redução } \\
\text { observada }\end{array}$ & $\mathrm{KW}=2,71 ; p=0,4391$ \\
\hline & $\begin{array}{l}\text { Valor Médio } \\
\text { das AIHs }\end{array}$ & 1548,17 & 4368,87 & 9249,99 & 19639,7 & aumento & $K W=140,94 ; p<0,0001$ \\
\hline & Qtd. AIHs & 985 & 126 & 21 & 1 & - & - \\
\hline
\end{tabular}


Tabela 1 (Continuação)

\begin{tabular}{|c|c|c|c|c|c|c|c|}
\hline \multirow{2}{*}{ CID } & \multirow{2}{*}{ Variável } & \multicolumn{4}{|c|}{ Terapia Dietética Utilizada } & \multirow{2}{*}{ Análise geral } & \multirow{2}{*}{ Kruskal-Wallis ANOVA } \\
\hline & & Nenhuma & NE & NP & NEP & & \\
\hline \multirow{4}{*}{$\begin{array}{l}\hat{U} \\
0 \\
0\end{array}$} & $\begin{array}{l}\text { Permanência } \\
\text { Média (dias) }\end{array}$ & 7,66 & 15,48 & 15,57 & - & aumento & $K W=29,54 ; p<0,0001$ \\
\hline & $\begin{array}{l}\text { Taxa Média de } \\
\text { Óbitos }\end{array}$ & 0,2231 & 0,1818 & 0,1429 & - & $\begin{array}{l}\text { redução } \\
\text { observada }\end{array}$ & $K W=0,96 ; p=0,6197$ \\
\hline & $\begin{array}{l}\text { Valor Médio } \\
\text { das AIHs }\end{array}$ & 1744,35 & 4306,03 & 5566,72 & - & aumento & $K W=59,71 ; p<0,0001$ \\
\hline & Qtd. AIHs & 251 & 33 & 21 & - & - & - \\
\hline \multirow{4}{*}{$\begin{array}{l}\stackrel{ }{U} \\
0 \\
0\end{array}$} & $\begin{array}{l}\text { Permanência } \\
\text { Média (dias) }\end{array}$ & 6,17 & 17,88 & 26,8 & 21,75 & aumento & $\mathrm{KW}=89,92 ; \mathrm{p}<0,0001$ \\
\hline & $\begin{array}{l}\text { Taxa Média de } \\
\text { Óbitos }\end{array}$ & 0,0494 & 0,2143 & 0,1 & 0,25 & aumento & $K W=35,58 ; p<0,0001$ \\
\hline & $\begin{array}{l}\text { Valor Médio } \\
\text { das AIHs }\end{array}$ & 2590,43 & 6688,72 & 10787,59 & 8152,45 & aumento & $K W=64 ; p<0,0001$ \\
\hline & Qtd. AIHs & 2166 & 56 & 10 & 8 & - & - \\
\hline
\end{tabular}

Nota: NE = Nutrição Enteral; NP = Nutrição Parenteral; NEP = Nutrição Enteral e Parenteral; KW = estatística Kruskal-Wallis; * a eventual redução não é considerada relevante devido ao pequeno número de internações.

Ao considerar o registro de ocorrência de óbito observado nas internações analisadas, os resultados revelaram a ocorrência de registros de taxa média de óbito inferior em pacientes submetidos às terapias dietéticas enterais e parenterais com diagnóstico de neoplasias malignas no esôfago (C15), no pâncreas (C25) e no intestino delgado (C17). Destaque é dado ao CID C15 que apresenta reduzida taxa de óbito nas internações que utilizaram nutrição enteral e diferenças significativas entre os grupos atestadas através do teste de Kruskal-Wallis.

A Tabela 2 mostra os resultados obtidos para as análises de avaliação comparativa do tempo médio de permanência, taxa média de óbitos e valor médio da internação para as internações que utilizaram UTI.

\section{Tabela 2}

Relação entre Indicadores Hospitalares (tempo de permanência, taxa de óbito e valor médio da internação) e o uso de Terapias Nutricionais Enterais e Parenterais em pacientes neoplásicos com idade igual ou superior a 12 anos que utilizaram internação em UTI em hospitais públicos do RS em 2017

\begin{tabular}{|c|c|c|c|c|c|c|c|}
\hline \multirow{2}{*}{ CID } & \multirow{2}{*}{ Variável } & \multicolumn{4}{|c|}{ Terapia Dietética Utilizada } & \multirow{2}{*}{ Análise geral } & \multirow{2}{*}{ Kruskal-Wallis ANOVA } \\
\hline & & Nenhuma & NE & NP & NEP & & \\
\hline \multirow{4}{*}{$\begin{array}{l}\boxminus \\
\dot{0} \\
\stackrel{0}{0} \\
0 \\
0\end{array}$} & $\begin{array}{l}\text { Permanência } \\
\text { Média (dias) }\end{array}$ & 11,99 & 19,41 & 21,51 & 25,88 & aumento & $K W=328,55 ; p<0,0001$ \\
\hline & $\begin{array}{l}\text { Taxa Média } \\
\text { de Óbitos }\end{array}$ & 0,2017 & 0,3326 & 0,1750 & 0,2647 & $\begin{array}{l}\text { redução ob- } \\
\text { servada }\end{array}$ & $\mathrm{KW}=71,43 ; \mathrm{p}<0,0001$ \\
\hline & $\begin{array}{l}\text { Valor Médio } \\
\text { das AIHs }\end{array}$ & 6115,88 & 9232,05 & 10057,95 & 11326,46 & aumento & $\mathrm{KW}=321,1 ; \mathrm{p}<0,0001$ \\
\hline & Qtd. AIHs & 2895 & 941 & 160 & 34 & - & - \\
\hline \multirow{4}{*}{$\begin{array}{l}n \\
ت \\
ن \\
0 \\
0\end{array}$} & $\begin{array}{l}\text { Permanência } \\
\text { Média (dias) }\end{array}$ & 10,15 & 20,17 & 24,14 & 46,83 & aumento & $\mathrm{KW}=34,35 ; \mathrm{p}<0,0001$ \\
\hline & $\begin{array}{l}\text { Taxa Média } \\
\text { de Óbitos }\end{array}$ & 0,3662 & 0,0714 & 0,2857 & 0,5000 & $\begin{array}{l}\text { redução ob- } \\
\text { servada }\end{array}$ & $\mathrm{KW}=22,4 ; \mathrm{p}<0,0001$ \\
\hline & $\begin{array}{l}\text { Valor Médio } \\
\text { das AIHs }\end{array}$ & 7346,40 & 12133,29 & 12623,42 & 12765,47 & aumento & $K W=26,67 ; p<0,0001$ \\
\hline & Qtd. AIHs & 71 & 84 & 14 & 6 & - & - \\
\hline
\end{tabular}


Tabela 2 (Continuação)

\begin{tabular}{|c|c|c|c|c|c|c|c|}
\hline \multirow{2}{*}{ CID } & \multirow{2}{*}{ Variável } & \multicolumn{4}{|c|}{ Terapia Dietética Utilizada } & \multirow{2}{*}{ Análise geral } & \multirow{2}{*}{ Kruskal-Wallis ANOVA } \\
\hline & & Nenhuma & NE & NP & NEP & & \\
\hline \multirow{4}{*}{$\begin{array}{l}\bullet \\
\cup \\
\cup \\
0 \\
\ddots\end{array}$} & $\begin{array}{l}\text { Permanência } \\
\text { Média (dias) }\end{array}$ & 12,58 & 16,21 & 21,77 & 20,50 & aumento & $K W=7,26 ; p=0,064$ \\
\hline & $\begin{array}{l}\text { Taxa Média } \\
\text { de Óbitos }\end{array}$ & 0,2568 & 0,2286 & 0,1538 & 0,5000 & $\begin{array}{l}\text { redução ob- } \\
\text { servada }\end{array}$ & $K W=1,43 ; p=0,6989$ \\
\hline & $\begin{array}{l}\text { Valor Médio } \\
\text { das AIHs }\end{array}$ & 7549,97 & 10111,48 & 8229,65 & 12682,09 & aumento & $K W=11,86 ; p=0,0079$ \\
\hline & Qtd. AIHs & 74 & 70 & 13 & 2 & - & - \\
\hline \multirow{4}{*}{$\begin{array}{l}\stackrel{J}{U} \\
\text { 光 }\end{array}$} & $\begin{array}{l}\text { Permanência } \\
\text { Média (dias) }\end{array}$ & 9,14 & 18,14 & 29,00 & - & aumento & $K W=40,96 ; p<0,0001$ \\
\hline & $\begin{array}{l}\text { Taxa Média } \\
\text { de Óbitos }\end{array}$ & 0,1429 & 0,5263 & 1,0000 & - & aumento & $K W=47,17 ; p<0,0001$ \\
\hline & $\begin{array}{l}\text { Valor Médio } \\
\text { das AIHs }\end{array}$ & 6091,00 & 7459,20 & 12925,84 & - & aumento & $\mathrm{KW}=6,15 ; \mathrm{p}=0,0462$ \\
\hline & Qtd. AIHs & 308 & 57 & 1 & - & - & - \\
\hline \multirow{4}{*}{$\begin{array}{l}\stackrel{-}{U} \\
0 \\
0\end{array}$} & $\begin{array}{l}\text { Permanência } \\
\text { Média (dias) }\end{array}$ & 13,44 & 15,48 & 1,00 & - & aumento * & $K W=6,65 ; p=0,036$ \\
\hline & $\begin{array}{l}\text { Taxa Média } \\
\text { de Óbitos }\end{array}$ & 0,0823 & 0,3163 & 0,0000 & - & aumento * & $K W=30,23 ; p<0,0001$ \\
\hline & $\begin{array}{l}\text { Valor Médio } \\
\text { das AIHs }\end{array}$ & 6361,91 & 8670,83 & 2066,90 & - & aumento * & $K W=32,69 ; p<0,0001$ \\
\hline & Qtd. AIHs & 243 & 98 & 1 & - & - & - \\
\hline \multirow{4}{*}{$\begin{array}{l}\stackrel{N}{U} \\
\text { Oㅂㅂ }\end{array}$} & $\begin{array}{l}\text { Permanência } \\
\text { Média (dias) }\end{array}$ & 14,61 & 18,31 & - & - & aumento & $K W=0,25 ; p=0,6164$ \\
\hline & $\begin{array}{l}\text { Taxa Média } \\
\text { de Óbitos }\end{array}$ & 0,2778 & 0,3077 & - & - & aumento & $K W=0,03 ; p=0,8586$ \\
\hline & $\begin{array}{l}\text { Valor Médio } \\
\text { das AIHs }\end{array}$ & 2932,92 & 7721,79 & - & - & aumento & $\mathrm{KW}=10 ; \mathrm{p}=0,0016$ \\
\hline & Qtd. AIHs & 18 & 13 & - & - & - & - \\
\hline \multirow{4}{*}{$\begin{array}{l}\stackrel{ }{U} \\
\text { U } \\
\text { 븐 }\end{array}$} & $\begin{array}{l}\text { Permanência } \\
\text { Média (dias) }\end{array}$ & 9,63 & 35,50 & - & 16,00 & aumento & $K W=6,13 ; p=0,0465$ \\
\hline & $\begin{array}{l}\text { Taxa Média } \\
\text { de Óbitos }\end{array}$ & 0,5000 & 0,5000 & - & 1,0000 & aumento & $\mathrm{KW}=0,89 ; \mathrm{p}=0,6412$ \\
\hline & $\begin{array}{l}\text { Valor Médio } \\
\text { das AIHs }\end{array}$ & 4214,93 & 12968,05 & - & 10189,37 & aumento & $\mathrm{KW}=4,04 ; p=0,1324$ \\
\hline & Qtd. AIHs & 8 & 8 & - & 1 & - & - \\
\hline \multirow{4}{*}{ 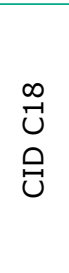 } & $\begin{array}{l}\text { Permanência } \\
\text { Média (dias) }\end{array}$ & 11,36 & 16,68 & 21,70 & 18,50 & aumento & $\mathrm{KW}=36,5 ; \mathrm{p}<0,0001$ \\
\hline & $\begin{array}{l}\text { Taxa Média } \\
\text { de Óbitos }\end{array}$ & 0,2690 & 0,4043 & 0,2162 & 0,1250 & $\begin{array}{l}\text { redução ob- } \\
\text { servada }\end{array}$ & $K W=5,48 ; p=0,1399$ \\
\hline & $\begin{array}{l}\text { Valor Médio } \\
\text { das AIHs }\end{array}$ & 6383,50 & 8614,42 & 10340,02 & 9209,94 & aumento & $K W=36,28 ; p<0,0001$ \\
\hline & Qtd. AIHs & 290 & 47 & 37 & 8 & - & - \\
\hline \multirow{4}{*}{$\begin{array}{l}\stackrel{\operatorname{Un}}{U} \\
0 \\
\stackrel{U}{U}\end{array}$} & $\begin{array}{l}\text { Permanência } \\
\text { Média (dias) }\end{array}$ & 13,62 & 23,94 & 21,94 & 13,00 & aumento * & $K W=13,97 ; p=0,0029$ \\
\hline & $\begin{array}{l}\text { Taxa Média } \\
\text { de Óbitos }\end{array}$ & 0,3571 & 0,0588 & 0,1176 & 0,0000 & $\begin{array}{l}\text { redução ob- } \\
\text { servada }\end{array}$ & $K W=13,59 ; p=0,0035$ \\
\hline & $\begin{array}{l}\text { Valor Médio } \\
\text { das AIHs }\end{array}$ & 7550,21 & 11340,30 & 10680,40 & 19639,70 & aumento & $K W=17,44 ; p=0,0006$ \\
\hline & Qtd. AIHs & 84 & 34 & 17 & 1 & - & - \\
\hline
\end{tabular}


Tabela 2 (Continuação)

\begin{tabular}{|c|c|c|c|c|c|c|c|}
\hline \multirow{2}{*}{ CID } & \multirow{2}{*}{ Variável } & \multicolumn{4}{|c|}{ Terapia Dietética Utilizada } & \multirow{2}{*}{ Análise geral } & \multirow{2}{*}{ Kruskal-Wallis ANOVA } \\
\hline & & Nenhuma & NE & NP & NEP & & \\
\hline \multirow{4}{*}{$\begin{array}{l}\text { 元 } \\
0 \\
0\end{array}$} & $\begin{array}{l}\text { Permanência } \\
\text { Média (dias) }\end{array}$ & 10,40 & 20,78 & 9,85 & - & $\begin{array}{l}\text { redução ob- } \\
\text { servada }\end{array}$ & $\mathrm{KW}=7,53 ; p=0,0232$ \\
\hline & $\begin{array}{l}\text { Taxa Média } \\
\text { de Óbitos }\end{array}$ & 0,4000 & 0,1111 & 0,0000 & - & $\begin{array}{l}\text { redução ob- } \\
\text { servada }\end{array}$ & $K W=8,94 ; p=0,0115$ \\
\hline & $\begin{array}{l}\text { Valor Médio } \\
\text { das AIHs }\end{array}$ & 5454,41 & 9394,33 & 6538,14 & - & aumento & $\mathrm{KW}=6,14 ; p=0,0465$ \\
\hline & Qtd. AIHs & 35 & 9 & 13 & - & - & - \\
\hline \multirow{4}{*}{$\begin{array}{l}\stackrel{ }{\cup} \\
0 \\
0\end{array}$} & $\begin{array}{l}\text { Permanência } \\
\text { Média (dias) }\end{array}$ & 12,12 & 35,77 & 32,40 & 18,80 & aumento & $K W=30,82 ; p<0,0001$ \\
\hline & $\begin{array}{l}\text { Taxa Média } \\
\text { de Óbitos }\end{array}$ & 0,1278 & 0,3846 & 0,0000 & 0,4000 & $\begin{array}{l}\text { redução ob- } \\
\text { servada }\end{array}$ & $K W=9,08 ; p=0,0282$ \\
\hline & $\begin{array}{l}\text { Valor Médio } \\
\text { das AIHs }\end{array}$ & 8419,06 & 14790,84 & 15439,44 & 11038,52 & aumento & $K W=26 ; p<0,0001$ \\
\hline & Qtd. AIHs & 133 & 13 & 5 & 5 & - & - \\
\hline
\end{tabular}

Nota: NE = Nutrição Enteral; NP = Nutrição Parenteral; NEP = Nutrição Enteral e Parenteral; KW = estatística Kruskal-Wallis; * a eventual redução não é considerada relevante devido ao pequeno número de internações.

Os resultados sugerem uma taxa média de mortalidade ligeiramente reduzida para as internações realizadas sob o diagnóstico principal associada ao CID Cap. II que utilizaram internação em UTI e procedimento de nutrição parenteral. Também é observada uma taxa de mortalidade reduzida para internações realizadas sob o diagnóstico principal de CID C15, C16 e C25 que utilizaram terapias de nutrição enteral ou parenteral. Destaque é dado aos CIDs C15, C25 e C17 que apresentam reduzida taxa de óbito nas internações que utilizaram nutrição enteral e/ou parenteral e diferenças significativas entre os grupos atestada através do teste de Kruskal-Wallis.

De modo geral, é observado que o tempo médio de permanência e o custo total médio das internações de pacientes submetidos a procedimentos de nutrição enteral e parenteral é superior ao dos pacientes que não utilizam tais terapias dietéticas. É razoável considerar que tal resultado se deve a possibilidade de que internações que utilizam terapias dietéticas enterais e/ou parenterais apresentem um quadro clínico mais grave, elevando o tempo médio de permanência do paciente e aumentando os custos totais das internações.

\section{DISCUSSÕES}

Os resultados deste estudo, no que tange ao tempo médio de permanência e valor médio das in- ternações, vão de encontro aos achados de alguns outros estudos conduzidos em âmbito internacional. Estudo que objetivou investigar os impactos de suplementos nutricionais orais (SNO) em pacientes hospitalares, utilizando dados do Premier Perspective Database, evidenciou que o uso de tais suplementos diminuiu o tempo de permanência em cerca de 2,3 dias, o custo em cerca de U\$ 4734 e o risco de readmissão hospitalar dos pacientes internados ${ }^{22}$. Outro estudo conduzido por meta-análise evidenciou que o uso de SNO reduziu significativamente a hospitalização, sugerindo ainda que o uso de SNO está associado a uma qualidade de vida melhorada, redução de infecções, redução de complicações pós-operatórias, redução de quedas e limitações funcionais ${ }^{23,24}$.

Entretanto, os resultados deste estudo corroboram outros achados anteriores no sentido de que o tempo de permanência de pacientes submetidos a procedimentos de terapia nutricional parenteral é superior à permanência de pacientes submetidos à nutrição enteral. De fato, estudo anterior mostrou que pacientes com câncer gastrointestinal submetidos a nutrição parenteral apresentaram maior ocorrência de complicações pós-operatórias do que os submetidos à nutrição enteral, sendo que o tempo médio de permanência no pós-operatório foi de 13,4 dias para pacientes submetidos à nutrição enteral e 15 dias para pacientes submetidos à nutrição parenteral ${ }^{25}$. 
A questão acerca dos potenciais benefícios associados ao uso de terapias nutricionais enterais e parenterais ainda é tema controverso. Estudo recente, conduzido em 44 hospitais da França, tendo como objetivo comparar a eficácia da nutrição enteral e parenteral precoce em pacientes com choque séptico e em ventilação mecânica, sugeriu que a nutrição enteral precoce não reduziu a mortalidade ou o risco de infecções secundárias nos pacientes, mas foi associada a um risco maior de complicações digestivas, em comparação com a parenteral ${ }^{26}$. Por outro lado, estudo sugere que a adoção de nutrição enteral, quando comparada à nutrição parenteral, está associada a um menor risco de complicações infecciosas, custo reduzido, reestabelecimento da função digestiva e duração reduzida da permanência ${ }^{27-29}$.

A nutrição enteral e parenteral é especialmente estratégica em casos de pacientes internados em UTIs ${ }^{7,12,30}$. Quanto a isso, os resultados deste estudo sugerem que a administração eficiente de terapias de nutrição enteral e parenteral tem potencial de reduzir a taxa de óbito em internações hospitalares com quadros clínicos mais graves e que necessitam de internação em UTIs realizadas sob o diagnóstico de neoplasias malignas.

Tendo como base tais resultados, este estudo evidencia que a discussão acerca da adoção de terapias dietéticas enterais e parenterais ainda carece de aprofundamento. De fato, tal questão permanece sendo tema controverso cujos achados de investigações e cientistas são considerados dispersos e difusos. Isso pode se dar pelo fato de que inúmeros fatores individuais e ambientais têm potencial de interferir na efetividade e consequências adversas da adoção de nutrição enteral e parenteral. Aspectos fisiológicos do paciente, a gravidade de seu caso, a natureza de sua doença, o risco infeccioso do ambiente hospitalar, a qualidade dos materiais hospitalares e insumos utilizados nas terapias dietéticas e outros inúmeros fatores podem impactar nos efeitos de terapias enterais e parenterais. Além disso, é necessário ainda considerar o impacto econômico na escolha do tipo de terapia dietética já que terapias parenterais apresentam custo significativamente superior ao de terapias enterais ${ }^{28}$.

Este estudo evidenciou ainda que o uso de terapias nutricionais enterais é muito superior ao uso de terapias parenterais. Tal evidência está de acordo com a literatura e os guidelines de práti- cas e protocolos médicos em função da preferência pelo uso da nutrição enteral, ao invés da parenteral, por ser mais fisiológica, menos invasiva, representando menor risco ao paciente, e por ter menor custo $^{31,32}$. De fato, a nutrição parenteral apresenta custo superior, risco mais elevado de infeção por ser procedimento mais invasivo. Logo, é preferível que profissionais da área médica optem pelo uso de terapias nutricionais que utilizem as vias digestivas naturais dos pacientes. De fato, os resultados deste estudo corroboram os achados de estudo anterior que evidencia que o uso de nutrição enteral apresenta resultados melhores com relação a taxa de mortalidade de pacientes neoplásicos do que o uso de nutrição parenteral ${ }^{33}$.

\section{CONCLUSÕES}

Este estudo evidenciou que pacientes diagnosticados com neoplasia maligna do pâncreas (C25), no intestino delgado (C17) ou no esôfago (C15) que foram internados em UTI e que receberam nutrição enteral e/ou parenteral apresentaram taxa de mortalidade significativamente inferior aos pacientes que não utilizaram nenhum suplemento nutricional.

Como limitação a ser apontada, este estudo considera apenas dados de internações hospitalares disponíveis no sistema de dados do SUS. Além disso, pelo fato de não ter sido considerado um perfil específico de pacientes internados faz com que os resultados apresentados não possam ser extrapolados para situações específicas. Também é necessário apontar que o estudo não considera o nível de adoção das terapias de nutrição enteral e parenteral assim como o nível de precocidade da adoção.

Cabe ainda sugerir que esforços por parte das instituições hospitalares e dos órgãos públicos regulamentadores de saúde no Brasil possam conduzir iniciativas no sentido de promover a elaboração e adoção de protocolos clínicos melhor estruturados e amplamente validados pelas instituições hospitalares no que tange à administração racional e eficiente de procedimentos de terapia nutricional enteral e parenteral. Além disso, são necessários estudos adicionais que considerem diagnósticos específicos, assim como estudos de custo-efetividade dos procedimentos de terapia nutricional enteral e parenteral. 


\section{CONFLITO DE INTERESSES}

O autor declara que não há conflito de interesses.

\section{FONTE DE FINANCIAMENTO}

Próprio.

\section{REFERÊNCIAS}

1. Somanchi M, Tao X, Mullin GE. The facilitated early enteral and dietary management effectiveness trial in hospitalized patients with malnutrition. J Parenter Enter Nutr 2011; 35: 209-216.

2. Waitzberg DL, Caiaffa WT, Correia MIT. Hospital malnutrition: the Brazilian national survey (IBRANUTRI): a study of 4000 patients. Nutrition 2001; 17: 573-580.

3. Dark DS, Pingleton SK. Nutrition and nutritional support in critically ill patients. J Intensive Care Med 1993; 8: 16-33.

4. Faisy C, Llerena MC, Savalle M, et al. Early ICU energy deficit is a risk factor for Staphylococcus aureus ventilator-associated pneumonia. Chest 2011; 140: 1254-1260.

5. DeFrances CJ, Lucas CA, Buie VC, et al. 2006 National hospital discharge survey. Natl Health Stat Rep 2008; 5: 1-20.

6. Lim SL, Ong KCB, Chan $\mathrm{YH}$, et al. Malnutrition and its impact on cost of hospitalization, length of stay, readmission and 3-year mortality. Clin Nutr 2012; 31: 345-350.

7. Singer $P$, Hiesmayr $M$, Biolo $G$, et al. Pragmatic approach to nutrition in the ICU: expert opinion regarding which calorie protein target. Clin Nutr 2014; 33: 246-251.

8. Braunschweig C, Gomez S, Sheean PM. Impact of Declines in Nutritional Status on Outcomes in Adult Patients Hospitalized for More Than 7 days. J Am Diet Assoc 2000; 100: 1316-1322.

9. Corkins MR, Guenter P, DiMaria-Ghalili RA, et al. Malnutrition diagnoses in hospitalized patients: United States, 2010. J Parenter Enter Nutr 2014; 38: 186-195.

10. Petros S, Horbach M, Seidel F, et al. Hypocaloric vs normocaloric nutrition in critically ill patients: a prospective randomized pilot trial. J Parenter Enter Nutr 2016; 40: 242-249.

11. Pichard C, Oshima T, Berger MM. Energy deficit is clinically relevant for critically ill patients: yes. Intensive Care Med 2015; 41: 335-338.

12. Singer $P$, Berger $M M$, Van den Berghe $G$, et al. ESPEN guidelines on parenteral nutrition: intensive care. Clin Nutr 2009; 28: 387-400.

13. Genton L, Dupertuis YM, Romand J-A, et al. Higher calorie prescription improves nutrient delivery during the first 5 days of enteral nutrition. Clin Nutr 2004; 23: 307-315.
14. Heyland DK, Dhaliwal R, Wang M, et al. The prevalence of iatrogenic underfeeding in the nutritionally 'at-risk'critically ill patient: Results of an international, multicenter, prospective study. Clin Nutr 2015; 34: 659-666.

15. Oshima $T$, Deutz NE, Doig G, et al. Protein-energy nutrition in the ICU is the power couple: a hypothesis forming analysis. Clin Nutr 2016; 35: 968-974.

16. Heidegger CP, Berger MM, Graf S, et al. Optimisation of energy provision with supplemental parenteral nutrition in critically ill patients: a randomised controlled clinical trial. The Lancet 2013; 381: 385-393.

17. Oshima T, Heidegger C-P, Pichard C. Supplemental parenteral nutrition is the key to prevent energy deficits in critically ill patients. Nutr Clin Pract 2016; 31: 432-437.

18. Evans RA, Strauss BJG. Cost and benefit of hospital, hospital-in-the-home and ambulatory care nutrition support services. Asia Pac J Clin Nutr 1998; 7: 241-244.

19. Gramlich L, Kichian K, Pinilla J, et al. Does enteral nutrition compared to parenteral nutrition result in better outcomes in critically ill adult patients? A systematic review of the literature. Nutrition 2004; 20: 843-848.

20. WHO. Cancer, https://www.who.int/news-room/fact-sheets/detail/cancer (2019, accessed 28 May 2019).

21. INCA. INCA - Instituto Nacional de Câncer. INCA - Instituto Nacional de Câncer, https://www.inca.gov.br/ (2019, accessed 28 May 2019).

22. Philipson TJ, Snider JT, Lakdawalla DN, et al. Impact of oral nutritional supplementation on hospital outcomes. Am J Manag Care 2013; 19: 121-128.

23. Elia M, Normand C, Laviano A, et al. A systematic review of the cost and cost effectiveness of using standard oral nutritional supplements in community and care home settings. Clin Nutr 2016; 35: 125-137.

24. Stratton RJ, Hebuterne X, Elia M. A systematic review and meta-analysis of the impact of oral nutritional supplements on hospital readmissions. Ageing Res Rev 2013; 12: 884-897.

25. Bozzetti F, Braga M, Gianotti L, et al. Postoperative enteral versus parenteral nutrition in malnourished patients with gastrointestinal cancer: a randomised multicentre trial. The Lancet 2001; 358: 1487-1492.

26. Reignier J, Boisramé-Helms J, Brisard L, et al. Enteral versus parenteral early nutrition in ventilated adults with shock: a randomised, controlled, multicentre, open-label, parallel-group study (NUTRIREA-2). The Lancet 2018; 391: 133-143.

27. Chow R, Bruera E, Chiu L, et al. Enteral and parenteral nutrition in cancer patients: a systematic review and meta-analysis. Ann Palliat Med 2016; 5: 30-41-41.

28. Seres DS, Valcarcel M, Guillaume A. Advantages of enteral nutrition over parenteral nutrition. Ther Adv Gastroenterol 2013; 6: 157-167.

29. Zhao X-F, Wu N, Zhao G-Q, et al. Enteral nutrition versus parenteral nutrition after major abdominal surgery 
in patients with gastrointestinal cancer: a systematic review and meta-analysis. J Investig Med 2016; 64: 1061-1074.

30. Kreymann KG, Berger MM, Deutz NEP, et al. ESPEN Guidelines on Enteral Nutrition: Intensive care. Clin Nutr 2006; 25: 210-223.

31. Oliveira E de V. Dieta em UTI, seu reflexo no tratamento, recuperaçäo e custo/dia do paciente internado. Aliment Nutr 1985; 6: 38-40.
32. Castrao DLL e, Freitas MM de, Zaban ALRS. Terapia nutricional enteral e parenteral: complicações em pacientes críticos - uma revisão de literatura. Comun Ciênc Saúde 2009; 20: 65-74.

33. Tamiya $H$, Yasunaga $H$, Matusi $H$, et al. Comparison of short-term mortality and morbidity between parenteral and enteral nutrition for adults without cancer: a propensity-matched analysis using a national inpatient database. Am J Clin Nutr 2015; 102: 1222-1228. 
